\title{
A Rare Cause of Dyspepsia: A Case Report of Gastric Diverticulum
}

Ren Yi Kow ${ }^{a}$, Dhauiddin Hai Ismail ${ }^{b}$, Chooi Leng Low ${ }^{c}, K^{\prime}$ hatrulnada Md Saad ${ }^{d,}$ Ed Simor Khan Mor Japar

$\mathrm{Khan}^{a}$

aDepartment of Orthopaedic, International Islamic University Malaysia, 25200 Kuantan, Pahang

bepartment of Surgery, Hospital Kuala Lipis, 27200 Kuala Lipis, Pahang

'Department of Radiology, Hospital Tengku Ampuan Afzan, 25100 Kuantan, Pahang

dDepartment of Radiology, Hospital Sultan Ahmad Shah, 28000 Temerloh, Pahang

${ }^{\mathrm{d} H o s p i t a l}$ Sultan Ahmad Shah, 28000 Temerloh, Pahang.

\section{ABSRACT}

A gastric diverticulum is an outpouching from the gastric mucosa. It is extremely rare. It is normally asymptomatic, but some may present with non-specific abdominal pain. A combination of upper gastrointestinal endoscopy and radiological contrast study such as oral barium study and computed tomography are needed to make a definite diagnosis and to rule out other associated pathology. Although treatment with medical therapy has been reported to be effective, the use of open and laparoscopic resection also yields a good outcome in the management of complicated gastric diverticulum. We present a case of symptomatic gastric diverticulum which has been successfully treated with medical therapy.

KEYWORDS : gastric, diverticulum, dyspepsia, medical, treatment

\section{INTRODUCTION}

A gastric diverticulum is defined as an outpouching from the gastric mucosa. ${ }^{1}$ It is extremely rare with a reported incidence range of $0.01-0.11 \%$ detected with upper gastrointestinal endoscopies and 0.04\% diagnosed via radiological studies. ${ }^{1}$ It is normally asymptomatic and found incidentally during radiological studies. Its symptoms, when present, are non-specific and can mimic a common gastritis. Here, we present a case of a symptomatic gastric diverticulum which is successfully managed conservatively.

\section{CASE REPORT}

Mrs $M$ is a 58-year-old housewife with no known medical illness. She complained of intermittent epigastric pain associated with nausea and vomiting for the past 2 years. Symptoms did not resolve with

Corresponding Author:

Dr Kow Ren Yi

Department of Orthopaedic

Hospital Kuala Lipis

27200 Kuala Lipis, Pahang

Email : renyi_kow@hotmail.com antacids prescribed by the general practitioner. She denied any constitutional symptoms and altered bowel habit. She is a passive smoker for 30 years. There is no family history of malignancy. Physical examination revealed a soft and non-tender abdomen. There was no palpable mass.

An oesophagogastroduodenalscope (OGDS) was performed to identify the cause of the dyspepsia. There was a diverticulum seen at the gastric fundus. There was no sign of perforation or bleeding. There was no other abnormality noted. Helicobacter pylori test was negative. Gastric biopsy was not taken.

A computed tomography scan of the abdomen was consistent with the diagnosis of gastric diverticulum. A well-defined saccular outpouching measuring $2.7 \mathrm{~cm} \times 2.4 \mathrm{~cm} \times 3.6 \mathrm{~cm}$ was noted on the posterior aspect of the gastric fundus. It was filled by iodinated oral contrast and was communicating with the gastric cavity.

She was started on a proton pump inhibitor after the OGDS. During the subsequent reviews at 3 and 6 months and 1 year, she claimed that her symptoms had subsided. 


\section{DISCUSSION}

Gastric diverticulum is first reported by Moebius in 1661 and later by Roax in $1774 .^{1}$ The literatures suggest that symptomatic gastric diverticula are normally found in patients aged between 20-60 years. ${ }^{1-2}$ It rarely $(4 \%)$ occurred in patient with age below 20 years. ${ }^{1-2}$ It is equally found in both males and females. ${ }^{1-2}$

Gastric diverticula are classified by Schmidt and Walters as true (congenital) diverticula and false (acquired) diverticula. ${ }^{2}$ True diverticula contain all layers of gastric wall. It is postulated that these congenital diverticula occur due to splitting of the longitudinal muscular fibres at the cardia level, leaving only circular muscles in the gastric wall and hence creating a weak point to allow the formation of diverticula during the foetal period. ${ }^{2}$ Foetal diverticula reported by Lewis in 1908 and then by Reich in 1941 further support this hypothesis. ${ }^{1-2}$ False diverticula, on the other hand, do not contain all layers of gastric wall. ${ }^{1-2}$ False diverticula can be classified into pulsation and traction diverticula based on the etiology. ${ }^{1-2}$ Pulsation diverticula occur due to increased intraluminal pressure secondary to chronic coughing, obesity or pregnancy. ${ }^{1-2}$ Traction diverticula arise from perigastric adhesion from concurrent diseases, such as peptic ulcer disease, pancreatitis, malignancy and cholecystitis. ${ }^{1-2}$ Most of the true diverticula (70\%) are found at the posterior wall of the stomach, just below the gastroesophageal junction. ${ }^{1}$

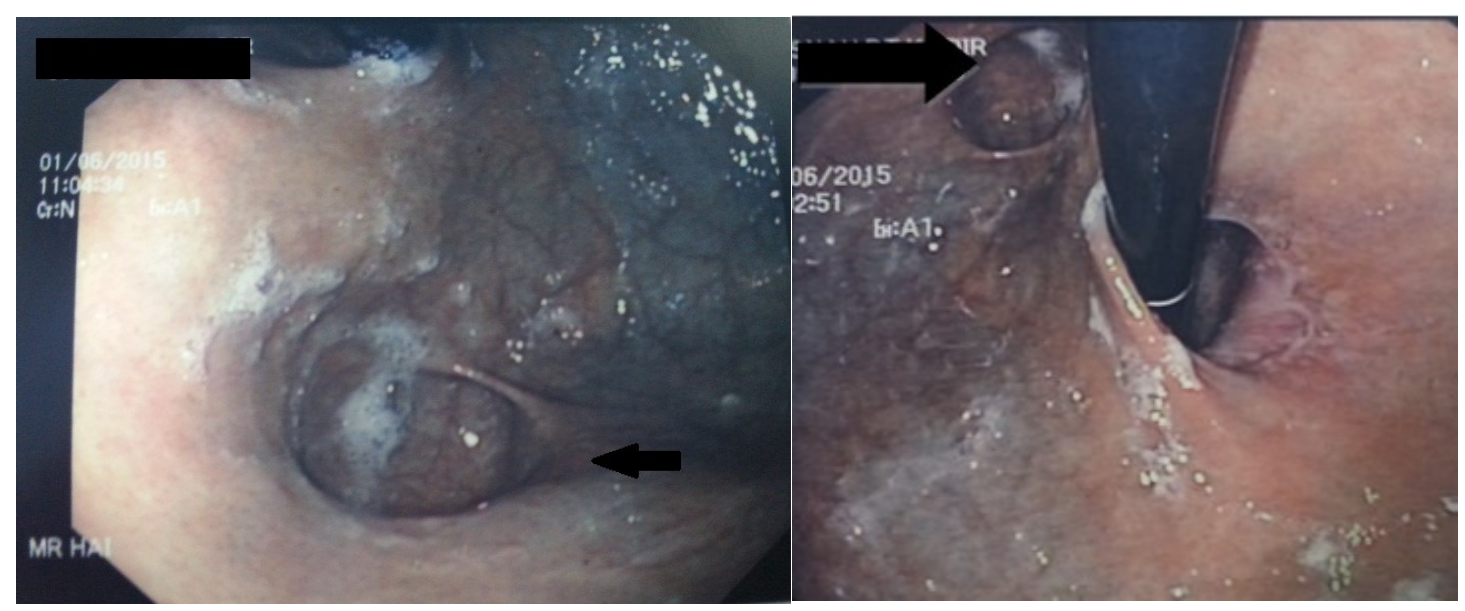

Figure 1 An endoscopic view of the gastric diverticulum located at the posterior fundus of the stomach (arrow). There is no sign of gastritis, perforation or bleeding.

Most of the gastric diverticula are asymptomatic. ${ }^{1}$ Its symptoms, when present, are vague abdominal or epigastric pain, nausea, vomiting and occasionally dysphagia $^{2}$ It is difficult to diagnose a gastric diverticulum. Most of them are incidental findings in radiological studies. ${ }^{3} \mathrm{~A}$ combination of oesophagogastroduodenoscopy (OGDS) and radiological contrast study such as oral barium study and computed tomography scans are needed to make a definite diagnosis and to rule out other associated pathology. ${ }^{3}$ In this case, a gastric diverticulum communicating with the gastric cavity is noted during an OGDS examination and is confirmed by a CT contrast study.

There is no guideline on the treatment of gastric diverticulum as this is rare. Both medical therapy and surgical resection have been reported to treat a gastric diverticulum. Asymptomatic gastric diverticula can be treated conservatively with a proton pump inhibitor (PPI) with regular follow-up and examination to identify the potential complications such as haemorrhage and perforation. ${ }^{1}$

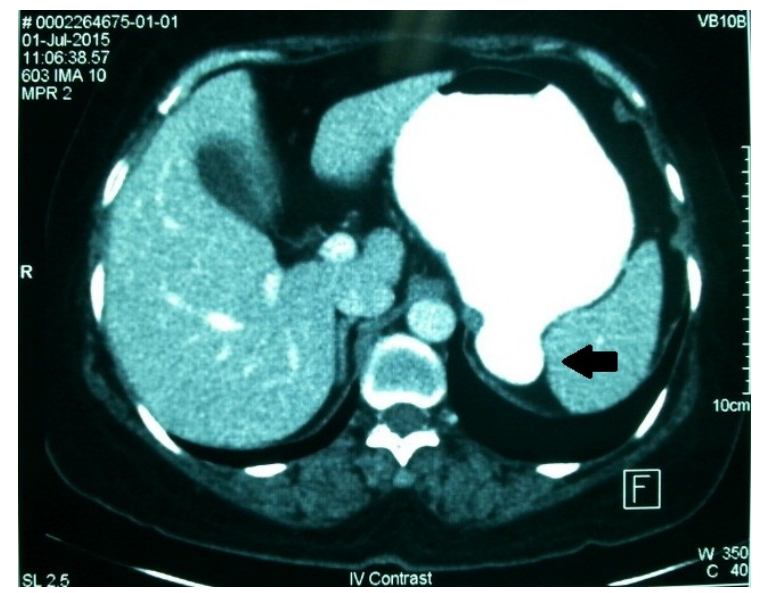

Figure 2 CT scan with contrast showed a well-defined saccular collection measuring $2.7 \mathrm{~cm} \times 2.4 \mathrm{~cm} \times 3.6 \mathrm{~cm}$ on the posterior fundus. It is communicating with the gastric cavity as evidenced by filling of iodinated oral contrast 
In this case, the patient was given a trial of PPI and her symptoms resolved with the medication. In $10 \%$ of the patients, surgery is required. ${ }^{4}$ The indications of surgical resection are large gastric diverticulum (more than $4 \mathrm{~cm}$ ), symptomatic and non-responsive to medical therapy, chronically inflamed, or complicated with ulceration, bleeding, perforation or malignancy. ${ }^{1,4}$ It should be noted that not all symptomatic gastric diverticula need to be resected. In one study, 30 of 49 symptomatic patients with gastric diverticulum, the symptoms were due to other gastrointestinal disease. ${ }^{4} \mathrm{~A}$ thorough investigations and planning is needed prior to gastric diverticula resection. Imaging such as computed tomography scan is helpful in identifying the location of the gastric diverticulum. It is very important to identify the gastric diverticulum preoperatively as the gastric diverticulum is often collapsed intra-operatively and hidden in the splenic bed. ${ }^{4}$ It has been reported that the wrong part of the stomach is resected in a patient with gastric diverticulum. ${ }^{4}$ In our patient, she response to the medical therapy, hence we propose that all patients with symptomatic diverticula should be started with medical therapy before pursuing the option of resection unless it is complicated with the complications described.

\section{CONCLUSION}

Gastric diverticulum is a rare stomach pathology. It is rarely symptomatic and its symptoms, when present, can mimic gastritis. Treatment of symptomatic gastric diverticula includes medical therapy and surgical resection.

\section{REFERENCES}

1. Luigi M, Gianmarco R, Raffaele P et al. Large symptomatic gastric diverticula: Two case reports and a brief review of literature. World J Gastroenterol 2013;19(36):6114-6117.

2. DuBois B, Powell B, Voeller G. Gastric Diverticulum:"A Wayside House of III Fame" with a Laparoscopic Solution. JSLS 2012 JulSep;16(3):473-477.

3. Tsitsias T, Finch JG. Gastric Diverticulum of the Prepyloric Region: A Rare Presentation of Gastric Diverticulum. Case Rep Gastroenterol 2012;6:150-154.

4. SC Donkervoort, LC Baak, JLG Blaauwgeers et al. Laparoscopic Resection of a Symptomatic
Gastric Diverticulum: a Minimally Invasive

Solution. JSLS 2006;10:525-527. 
